\title{
Effects of Hemodialysis on Intraocular Pressure and Ocular Biological Parameters in Different Angle Structures
}

\author{
Fenglei Wang $(\mathbb{D}$, Ling Wang $\mathbb{D}$, Zhiying Yu $\mathbb{D}$, Nan Chen $\mathbb{D}$, and Dabo Wang $\mathbb{1}$ \\ Ophthalmology, The Affiliated Hospital of Qingdao University, 1677, Wutaishan Road, 266000 Huangdao Region, Qingdao, China
}

Correspondence should be addressed to Ling Wang; tsingtaowl@hotmail.com

Received 30 September 2021; Accepted 2 February 2022; Published 12 February 2022

Academic Editor: Serena Fragiotta

Copyright (C) 2022 Fenglei Wang et al. This is an open access article distributed under the Creative Commons Attribution License, which permits unrestricted use, distribution, and reproduction in any medium, provided the original work is properly cited.

\begin{abstract}
Purpose. This study is aimed at evaluating the effects of hemodialysis on intraocular pressure (IOP) and exploring the possible factors affecting IOP. Methods. Fifty-two patients with hemodialysis (HD) that were diagnosed with chronic renal failure by nephrology were divided into four groups: wide angle, narrow angle, extremely narrow angle, and closed angle. IOP, central anterior chamber depth (ACD), lens thickness (LT), angle opening distance (AOD), trabecular-iris angle (TIA), iris thickness (IT), and ciliary body thickness (CBT) were recorded before and after HD. The Pearson coefficient test was used to determine correlations among changes in IOP and AOD, ACD, TIA, IT, CBT, and LT. Results. The IOP in the extremely narrow angle group had significant difference compared with that in the wide angle group and narrow angle group $(P<0.05, P<0.01)$. In the narrow angle group, change in LT was positively correlated with change in IOP $(P<0.05)$. In the extremely narrow angle group, change in LT was positively correlated with change in IOP $(\mathrm{P}<0.01)$, whereas changes in AOD and TIA were negatively correlated with change in IOP $(P<0.01 ; P<0.05)$. Conclusion. The effect of HD on IOP varies with the structure of the anterior chamber. The increasing of IOP in the extremely narrow-angle group is related with the changes of structure of anterior chamber.
\end{abstract}

\section{Introduction}

Hemodialysis is one of the renal replacement therapy methods used in patients with chronic renal failure. It involves the exchange of substances via diffusion or convection, which removes metabolic waste products from the body and corrects the water electrolyte balance. However, some patients may experience symptoms such as blurred vision, eye pain, and headache during or after dialysis $[1,2]$. Whether elevated intraocular pressure during long-term dialysis induces glaucoma or other ocular complications in patients on chronic maintenance hemodialysis has attracted the attention of ophthalmologists and prompted relevant research.

In some studies, an elevated IOP was measured after hemodialysis [3, 4], while others found no significant changes or even a decreased IOP [5-8]. The reasons for these differ- ences are unclear and may be related to the patient selection criteria utilized and factors influencing changes in IOP. There is currently little evidence available to draw upon to clarify whether this is related to the configuration of the anterior chamber angle.

It is known that chronic renal failure patients have insufficient local blood supply to the eyes [5], and fluctuations in IOP caused by hemodialysis may aggravate this condition or even cause severe, irreversible ischemic, and hypoxic damage to the optic nerve and retina. Therefore, it is necessary to analyze changes in IOP during hemodialysis and investigate related mechanisms to protect visual function in maintenance hemodialysis patients. The primary aims of the current study were to identify parameters associated with a high risk of visual function endangerment in maintenance hemodialysis patients. 


\section{Materials and Methods}

\subsection{Materials}

2.1.1. Study Population. Fifty-two eyes in 52 patients ( 25 male and 27 female) diagnosed with chronic renal failure and undergoing maintenance hemodialysis in the nephrology department of our hospital from January to December 2015 were enrolled in the current study. Informed consent was obtained from all individual participants included in the study. General characteristics of patients are summarized in Table 1. Only the right eyes of the patients were examined.

\subsubsection{Inclusion and Exclusion Criteria. Inclusion criteria were as follows:}

(1) Patients who exhibited good compliance and appropriate levels of physical activity (2-5 hours per week, such as walking, jogging, etc.) and had signed informed consents, (2) patients whose visual acuity was $\geq 0.3$ on the LogMAR scale, and (3) patients whose IOP was 10-21 mmHg

Exclusion criteria were as follows:

(1) Patients with a history of glaucoma or ocular hypertension or eye surgery, (2) patients suffering from concomitant vitreoretinal disorders, (3) patients exhibiting media opacity that affects optical clarity, and (4) patients diagnosed with diabetes

Patients were allocated to one of four groups based on the Shaffer grading system [9], which considers the width of the angle formed by the imaginary tangents of the cornea-trabecular meshwork and the anterior surface of the iris (i.e., the angle of the iris recess serves as the classification criterion) and ultrasound biomicroscopy examinations of which 3 or more quadrants met the reference standards. More specifically, patients with 3 or 4 quadrants with an open angle of $>20$ degrees were allocated to the wide-angle group $(n=22)$, patients with 3 or 4 quadrants with an open angle of 11-20 degrees were allocated to the narrow-angle group $(n=18)$, patients with 3 or 4 quadrants with an open angle of 0-10 degrees were allocated to the extremely narrow-angle group $(n=12)$, and patients with 3 or 4 quadrants with an open angle of 0 degrees were allocated to the closed-angle group $(n=0)$.

2.2. Methods. Hemodialysis patients were allocated to a wideangle group (WA group), a narrow-angle group (NA group), an extremely narrow-angle group (ENA group), or a closedangle group (CA group) based on their angular anatomy. Changes in IOP before and after HD were monitored in each group. Changes in anterior chamber parameters were investigated, and correlations between changes in IOP and changes in anterior chamber parameters were assessed.

2.2.1. Hemodialysis Method. Only morning session HD patients were included. All patients underwent $4 \mathrm{~h} \mathrm{HD}$ sessions, 3 days per week, at a blood-flow rate of $250 \mathrm{~mL} / \mathrm{min}$. Patients were treated using high-performance dialyzers: 4008S-type Fresenius HD machine (Germany) and a Campbell $8 \mathrm{~L}$ reusable dialyzer (Sweden); patient blood was dia-
TABLE 1: General characteristics of the patients.

\begin{tabular}{lccc}
\hline Variable & Mean & SD & Range \\
\hline Age (years) & 52.4 & 10.1 & $40-68$ \\
Hemodialysis duration (year) & 6.1 & 1.9 & $4-10$ \\
Total amount of ultrafiltration (mL) & 2318 & 612.3 & $1500-3100$ \\
Weight loss (kg) & 2.10 & 0.91 & $0.95-2.95$ \\
\hline
\end{tabular}

lyzed against bicarbonate dialysate $(1.5 \mathrm{mmol} / \mathrm{L}$ calcium $)$. All patients exhibited arteriovenous fistulae and used a polysulfone hollow-fiber dialyzer (Fx80; Germany). The total body weight was measured before and after HD.

2.2.2. Measurement of Plasma Osmotic Pressure. Blood samples were obtained within 60 seconds before the start and end of HD and then used to calculate plasma osmotic pressure (plasma osmotic pressure $=1.86 \times \mathrm{Na}+$ glucose $\div 18+$ urea nitrogen $\div 2+9$ ) .

2.2.3. Measurement of IOP. IOP was measured $30 \mathrm{~min}$ before the start of $\mathrm{HD}, 2 \mathrm{~h}$ after initiation of HD, and $30 \mathrm{~min}$ after ending HD, using a hand-held rebound tonometer (Suowei Rebound tonometer SW-500, Tianjin, China). Three consecutive measurements were averaged to obtain a mean IOP value.

\subsubsection{A-Ultrasound and Ultrasound Biomicroscopy (UBM)} Measurements. Patients were placed in the supine position. After administration of a topical anesthetic $(0.25 \%$ oxybuprocaine eye drop), the patients were allowed to fix their fingers to maintain vision straight ahead. Then, central anterior chamber depth and lens thickness were measured by A-ultrasonography (Quantel Medical, Ltd., Model Aviso, France), 30 min before HD and after ending HD. Ten measurements were performed, and the average value was used.

Patients were placed in the supine position, with constant indoor brightness. The UBM examinations were performed by one trained technician who used the UBM (Suowei Panoramic Biological Microscope SW-3200 L, Tianjin, China) with a $50 \mathrm{MHz}$ transducer probe. A total of 30 min before the start and end of hemodialysis, angle opening distance (AOD), trabecular-iris angle (TIA), iris thickness (IT), and ciliary body thickness (CBT) were measured by UBM at 12 o'clock, 6 o'clock, 3 o'clock, and 9 o'clock positions. All measurements were performed by the same examiner to minimize the bias, and the average values of the four quadrants were used:

(1) AOD: a straight line was drawn perpendicular to the corneal endothelium $500 \mu \mathrm{m}$ anterior to the scleral spur to the plane of the trabecular mesh, to the corresponding frontal surface of the iris. The length of this line was deemed to be the angle opening distance (Figure 1)

(2) TIA: a triangle with the AOD as a base and the recess at the iris root as a vertex and the angle between the vertices was the trabecular-iris angle (Figure 1) 


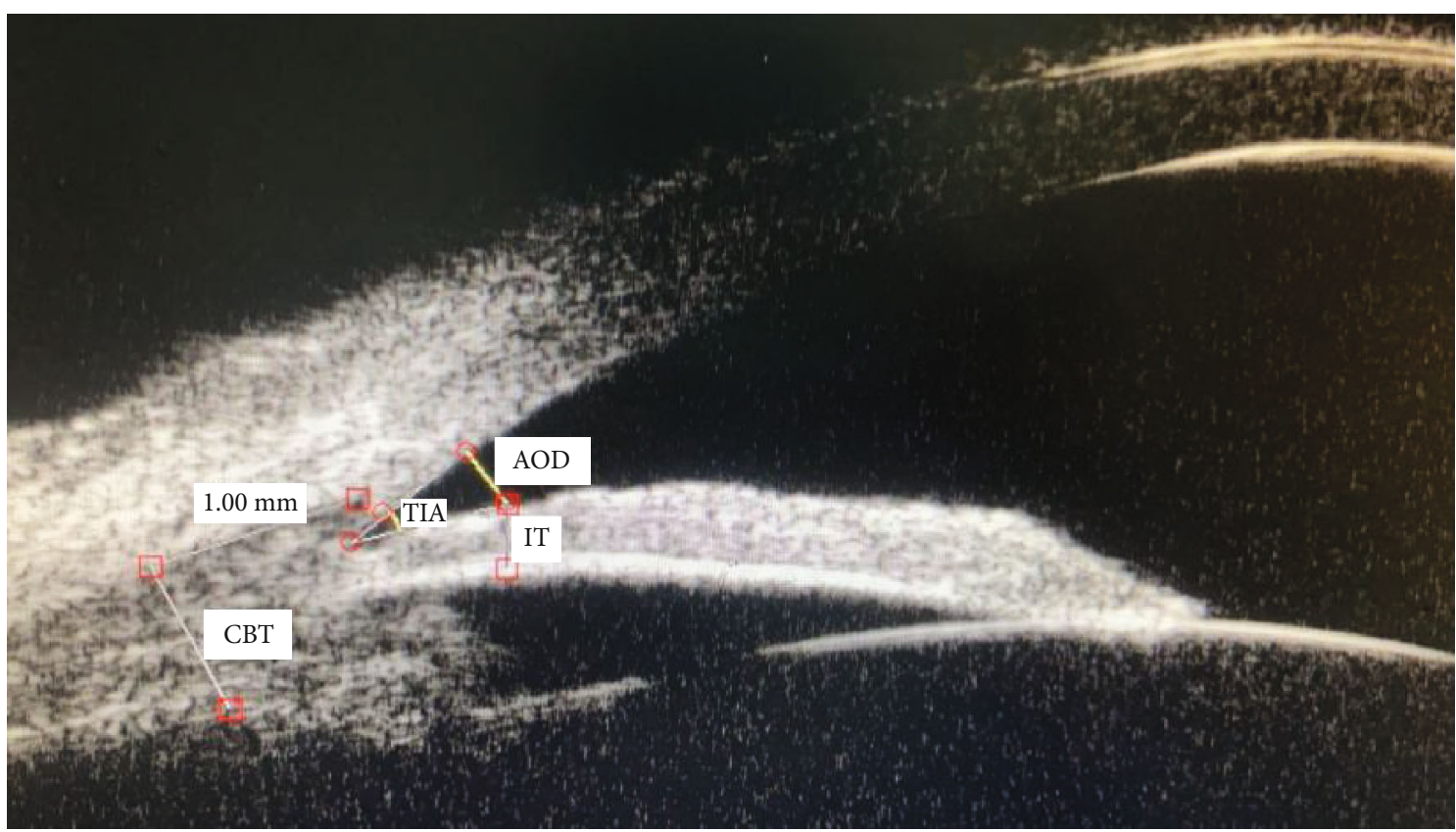

Figure 1: The measurements of anterior chamber parameters by ultrasound biomicroscopy. (AOD was $0.320 \mathrm{~mm}$, TIA was $25.0 \mathrm{D}$, IT was $0.35 \mathrm{~mm}$, and CBT was $0.81 \mathrm{~mm}$.)

TABLE 2: Effect of hemodialysis on plasma osmotic pressure and blood biochemical parameters $(\bar{x} \pm s)$.

\begin{tabular}{lcc}
\hline Variable & Pre-HD & Post-HD \\
\hline Urea nitrogen $(\mathrm{mmol} / \mathrm{l})$ & $26.06 \pm 5.48$ & $8.92 \pm 3.79$ \\
Glucose $(\mathrm{mmol} / \mathrm{l})$ & $5.96 \pm 1.19$ & $7.85 \pm 3.25$ \\
$\mathrm{Na}+(\mathrm{mmol} / \mathrm{l})$ & $138.18 \pm 2.40$ & $137.98 \pm 4.35$ \\
Creatinine $(\mu \mathrm{mol} / \mathrm{l})$ & $909.22 \pm 119.93$ & $395.52 \pm 118.92$ \\
Plasma osmotic pressure $\left(\mathrm{mOsm} / \mathrm{kgH}_{2} \mathrm{O}\right)$ & $279.37 \pm 6.16$ & $270.54 \pm 7.32$ \\
\hline
\end{tabular}

(3) IT: the iris thickness was traced at a specific point. The exact location was determined tracing a straight line perpendicular to the trabecular meshwork, $500 \mu \mathrm{m}$ in front of the scleral spur, which intersect the anterior surface of the iris (Figure 1)

(4) CBT: the thickness of ciliary body at the posterior $1 \mathrm{~mm}$ of the scleral spur (Figure 1)

2.3. Statistical Analysis. All data were analyzed using the SPSS statistical package, version 19.0. The Kolmogorov-Smirnov test was used to assess normality of the data. Variables comparisons in each group were performed utilizing the pairedsample $t$-test. IOP among groups was compared using the repeated measures one-way analysis of variance (ANOVA). The Pearson coefficient test was used to determine correlations among changes in IOP and AOD, ACD, TIA, IT, CBT, and LT. $P<0.05$ was considered to be statistically significant.

\section{Results}

3.1. Change in Plasma Osmotic Pressure. Plasma osmotic pressure after HD was significantly lower than plasma osmotic pressure before HD $(P<0.05, t=3.041)$, as shown in Table 2 .
3.2. Change in IOP. Changes in IOP in each group $2 \mathrm{~h}$ after the initiation of hemodialysis and $30 \mathrm{~min}$ after the end of hemodialysis are shown in Table 3.

\subsection{The Main Effect of Treatment and Time}

(1) Mean IOP differed significantly at different time points during hemodialysis $(F=41.69, P<0.01)$. The difference between IOP before hemodialysis and $2 \mathrm{~h}$ after the initiation of hemodialysis was statistically significant $(P<0.01)$, as was the difference between IOP $2 \mathrm{~h}$ after hemodialysis initiation and $30 \mathrm{~min}$ after the end of hemodialysis $(P<0.01)$

(2) Mean IOP differed significantly in different groups of hemodialysis patients $(F=6.44, P<0.01)$. IOP in the wide-angle group differed significantly from that in the extremely narrow-angle group $(P<0.05)$, and IOP in the narrow-angle group differed significantly from that in the extremely narrow-angle group $(P<0.01)$ (Table 3$)$

(3) The extremely narrow-angle group had significantly increased IOP $2 \mathrm{~h}$ after the initiation of hemodialysis 
TABLE 3: Intraocular pressure before hemodialysis, after $2 \mathrm{~h}$ of dialysis, and $30 \mathrm{~min}$ after the end of dialysis $(\bar{x} \pm s, \mathrm{mmHg})$.

\begin{tabular}{lcccc}
\hline Group & $n$ & Pre-HD & $2 \mathrm{~h}$ of HD & Post-HD \\
\hline WA group & 22 & $18.7 \pm 3.8$ & $18.1 \pm 4.9$ & $19.7 \pm 5.4$ \\
NA group & 18 & $17.0 \pm 4.4$ & $18.6 \pm 5.7$ & $18.7 \pm 6.1$ \\
ENA group & 12 & $17.0 \pm 4.7$ & $20.6 \pm 4.0$ & $18.4 \pm 3.8$ \\
\hline
\end{tabular}

$(P<0.05)$, and in that group IOP returned to prehemodialysis levels within $30 \mathrm{~min}$ after the end of hemodialysis $(P>0.05)$ (Figure 2$)$

\subsection{Correlations between Changes in Anterior Chamber} Parameters and IOP. The ciliary body was thinner in all groups within $30 \mathrm{~min}$ after the end of hemodialysis, but it was only statistically significantly thinner than before hemodialysis in the wide-angle group $(t=2.61, P<0.05)$. After hemodialysis, central anterior chamber depth, angle opening distance, and trabecular-iris angle were all smaller in all groups. The root of the iris became thinner, and the lens became thicker, but these differences were not statistically significant $(P>0.05)$ (Tables 4-6).

In the wide-angle group, there were no significant correlations between changes in anterior chamber parameters and the change in IOP. In the narrow-angle group, the change in lens thickness was positively correlated with the change in IOP. In the extremely narrow-angle group, the change in lens thickness was positively correlated with the change in IOP, whereas the changes in AOD and TIA were negatively correlated with the change in IOP (Tables 4-6; Figures 3-5).

\section{Discussion}

The effects of hemodialysis on IOP have been studied by many researchers in recent years. There are many different views on the relationship between hemodialysis and IOP, probably due to differences in research methods, hemodialysis parameters, selected subjects, and measuring time. Most researchers have observed increased IOP after hemodialysis. In 1964, Sitprija et al. [10, 11] reported the earliest study investigating relationships between hemodialysis and changes in IOP. They reported that hemodialysis could lead to an increase in IOP in animal models and uremia patients, with an average increase of $4-8 \mathrm{mmHg}$. They also reported that IOP increased by an average of $5.9 \mathrm{mmHg}$ in the first 3 hours of hemodialysis. In a study by De Marchi et al. [12], IOP increased significantly in 10 patients with narrow anterior chamber angles during hemodialysis $(7.8-12.5 \mathrm{mmHg})$. Some researchers have reported that IOP did not change over the entire duration of hemodialysis. In a study by Hojs and Pahor [13], there was no statistically significant difference between IOP before and after hemodialysis. A small number of researchers have reported reductions in IOP after hemodialysis. Gutmann and Vaziri [14] investigated IOP in hemodialysis patients and normal controls and reported that IOP was significantly reduced in hemodialysis patients, especially during the first 2 hours, but there was a slight increase at the

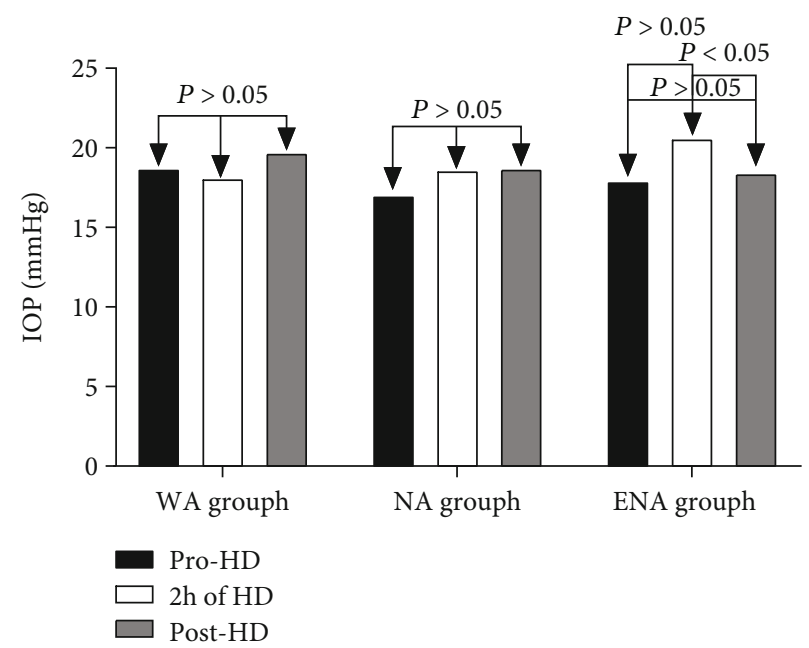

FIgURE 2: Intraocular pressure before hemodialysis, after $2 \mathrm{~h}$ of dialysis, and $30 \mathrm{~min}$ after the end of dialysis in three groups.

end of hemodialysis. In a study by Dinc et al. [15] involving 33 patients on hemodialysis, IOP decreased by approximately $1.3 \mathrm{mmHg}$ after hemodialysis. Yang et al. [16] investigated changes in IOP after hemodialysis in 34 patients with chronic renal failure and reported that mean IOP before hemodialysis was $15.1 \pm 2.6 \mathrm{mmHg}$, and it decreased to $13.9 \pm 2.2 \mathrm{mmHg}$ after hemodialysis. Cecchin et al. [4] found patients with elevated IOP after hemodialysis have a narrow anterior chamber angle, but there have been no relevant studies on changes in this narrow angle.

In the current study, IOP was significantly increased after 2 hours of hemodialysis in the extremely narrowangle group, whereas in the wide-angle group and the narrow-angle group, IOP was not significantly increased after 2 hours of hemodialysis. Our results suggest that there may be a dichotomous explanation for the IOP fluctuations. During the dialysis process, toxic substances in the blood (including urea, nitrogen, and creatinine) diffuse into the dialysate, leading to a significant decrease in plasma osmotic pressure [17-19], just as our study showed. And this will increase the aqueous humor production, which can increase IOP. Urea nitrogen concentration in the lens cannot decrease as rapidly as in blood during hemodialysis, resulting in an osmotic pressure imbalance between the lens and the aqueous humor. The lens absorbs water and expands, along with a reduced anterior chamber depth and a narrow angle leading to a poor outflow, and hence IOP also increases $[20,21]$. The counterpart is that dialysis can induce dehydration and ultrafiltration, leading to a thinning of the iris and ciliary body with widening the anterior chamber [22]. Consequently, increasing aqueous humor drainage can reduce IOP. Thus, there are many factors that can increase and decrease IOP during hemodialysis.

In this study, despite the increases in aqueous humor production in the wide-angle and narrow-angle groups, the compensatory capacity of the aqueous humor drainage pathway was relatively high, aqueous humor production and drainage were in equilibrium, and fluctuations in IOP during 
TABLE 4: Changes in anterior chamber parameters after hemodialysis in the wide-angle group and correlations with changes in IOP.

\begin{tabular}{|c|c|c|c|c|c|}
\hline \multirow{2}{*}{ Variable } & \multirow{2}{*}{ Pre-HD } & \multirow{2}{*}{ Post-HD } & \multirow{2}{*}{$P$ value } & \multicolumn{2}{|c|}{ Correlation* ${ }^{*}$} \\
\hline & & & & $r$ & $P$ value \\
\hline IOP (mmHg) & $18.7 \pm 3.8$ & $19.7 \pm 5.4$ & 0.224 & & \\
\hline $\mathrm{AOD}(\mathrm{mm})$ & $0.325 \pm 0.077$ & $0.321 \pm 0.073$ & 0.609 & 0.186 & 0.506 \\
\hline $\mathrm{ACD}(\mathrm{mm})$ & $2.87 \pm 0.31$ & $2.84 \pm 0.26$ & 0.415 & -0.008 & 0.976 \\
\hline TIA (D) & $27.86 \pm 5.78$ & $27.55 \pm 5.71$ & 0.697 & 0.466 & 0.080 \\
\hline IT (mm) & $0.425 \pm 0.066$ & $0.367 \pm 0.055$ & 0.393 & 0.017 & 0.952 \\
\hline $\mathrm{CBT}(\mathrm{mm})$ & $0.707 \pm 0.040$ & $0.688 \pm 0.044$ & 0.021 & 0.129 & 0.647 \\
\hline $\mathrm{LT}(\mathrm{mm})$ & $4.85 \pm 0.33$ & $4.86 \pm 0.42$ & 0.816 & -0.296 & 0.285 \\
\hline
\end{tabular}

${ }^{*}$ Correlation between changes in intraocular pressure and other variables.

TABLE 5: Changes in anterior chamber parameters after hemodialysis in the narrow-angle group and correlations with changes in IOP.

\begin{tabular}{|c|c|c|c|c|c|}
\hline \multirow{2}{*}{ Variable } & \multirow{2}{*}{ Pre-HD } & \multirow{2}{*}{ Post-HD } & \multirow{2}{*}{$P$ value } & \multicolumn{2}{|c|}{ Correlation* } \\
\hline & & & & $r$ & $P$ value \\
\hline IOP (mmHg) & $17.0 \pm 4.4$ & $18.7 \pm 6.1$ & 0.250 & & \\
\hline $\mathrm{AOD}(\mathrm{mm})$ & $0.225 \pm 0.052$ & $0.206 \pm 0.058$ & 0.144 & -0.057 & 0.853 \\
\hline $\mathrm{ACD}(\mathrm{mm})$ & $2.69 \pm 0.24$ & $2.59 \pm 0.27$ & 0.051 & -0.049 & 0.874 \\
\hline TIA (D) & $16.84 \pm 3.08$ & $15.22 \pm 3.66$ & 0.150 & -0.361 & 0.226 \\
\hline IT (mm) & $0.353 \pm 0.056$ & $0.350 \pm 0.052$ & 0.494 & -0.282 & 0.351 \\
\hline CBT (mm) & $0.669 \pm 0.064$ & $0.659 \pm 0.067$ & 0.218 & 0.444 & 0.129 \\
\hline $\mathrm{LT}(\mathrm{mm})$ & $4.96 \pm 0.53$ & $5.04 \pm 0.52$ & 0.121 & 0.610 & 0.027 \\
\hline
\end{tabular}

${ }^{*}$ Correlation between changes in intraocular pressure and other variables.

TABLE 6: Changes in anterior chamber parameters after hemodialysis in the extremely narrow-angle group and correlations with changes in IOP.

\begin{tabular}{|c|c|c|c|c|c|}
\hline \multirow{2}{*}{ Variable } & \multirow{2}{*}{ Pre-HD } & \multirow{2}{*}{ Post-HD } & \multirow{2}{*}{$P$ value } & \multicolumn{2}{|c|}{ Correlation* } \\
\hline & & & & $r$ & $P$ value \\
\hline IOP (mmHg) & $17.0 \pm 4.7$ & $18.4 \pm 3.8$ & 0.302 & & \\
\hline $\mathrm{AOD}(\mathrm{mm})$ & $0.134 \pm 0.064$ & $0.124 \pm 0.060$ & 0.532 & -0.845 & 0.004 \\
\hline $\mathrm{ACD}(\mathrm{mm})$ & $2.65 \pm 0.15$ & $2.59 \pm 0.16$ & 0.338 & -0.130 & 0.738 \\
\hline TIA (D) & $8.09 \pm 1.55$ & $7.52 \pm 1.67$ & 0.705 & -0.753 & 0.019 \\
\hline IT (mm) & $0.425 \pm 0.066$ & $0.411 \pm 0.070$ & 0.293 & 0.388 & 0.302 \\
\hline CBT (mm) & $0.669 \pm 0.064$ & $0.648 \pm 0.057$ & 0.088 & -0.218 & 0.574 \\
\hline $\mathrm{LT}(\mathrm{mm})$ & $4.72 \pm 0.31$ & $4.78 \pm 0.28$ & 0.334 & 0.820 & 0.007 \\
\hline
\end{tabular}

${ }^{*}$ Correlation between changes in intraocular pressure and other variables.

and after hemodialysis were not significant. However, aqueous humor drainage is low in patients with a shallow anterior chamber and a narrow anterior chamber angle [23, 24]. As plasma osmotic pressure decreases after hemodialysis, increased aqueous humor secretion may lead to an increase in IOP and may even cause acute angle-closure glaucoma. Therefore, compared with the other groups, the IOP of 2 hours after initiation of $\mathrm{HD}$ was significantly higher than that before hemodialysis in the extremely narrow-angle group.
Notably, $30 \mathrm{~min}$ after the end of hemodialysis, IOP had returned to prehemodialysis levels, even in the extremely narrow-angle group. Because in this group, the chamber angle was not completely closed, and the observed fluctuations in IOP during hemodialysis could recover after hemodialysis. An acute increase in IOP is highly likely in patients with complete angle closure [23]. There were no patients with complete angle closure among the 52 cases in the study, which may explain why no acute increases in IOP during hemodialysis were observed. Therefore, we believe that the 


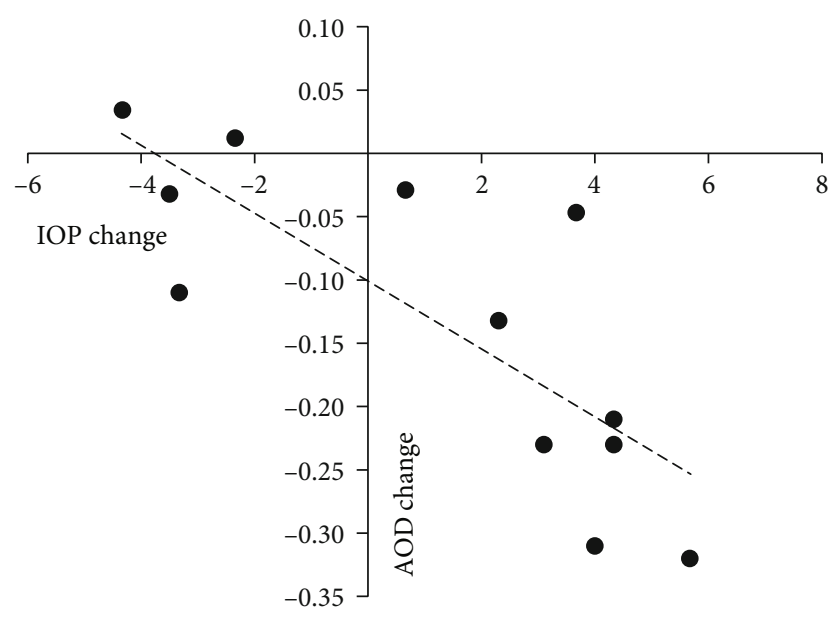

Figure 3: Change in AOD plotted against change in IOP in the extremely narrow-angle group. A significant correlation was found $(r=-0.845, P=0.004)$.

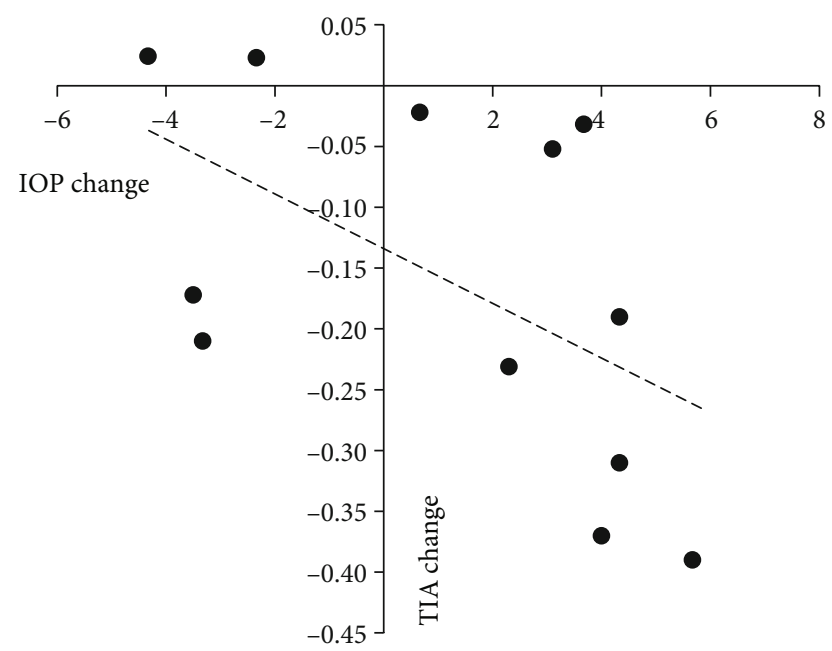

Figure 4: Change in TIA plotted against change in IOP in the extremely narrow-angle group. A significant correlation was found $(r=-0.753, P=0.019)$.

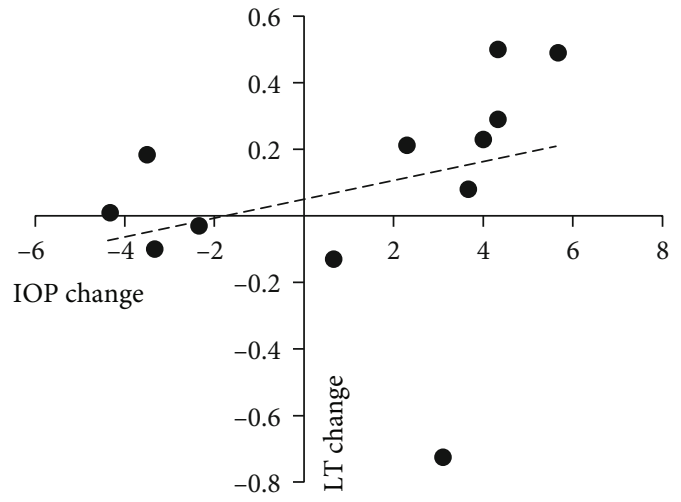

FIgure 5: Change in LT plotted against change in IOP in the extremely narrow-angle group. A significant correlation was found $(r=0.820, P=0.007)$. change in IOP during hemodialysis in patients with chronic renal failure is related to anterior chamber angle structure. An extremely narrow angle is a risk factor for elevated IOP during hemodialysis, while wide-angle patients are relatively safe. Our findings confirmed the importance of anterior chamber status by analyzing the relationship between anterior chamber parameters and IOP changes after hemodialysis. In the extremely narrow-angle group, lens thickening was associated with increasing IOP, whereas changes in AOD and TIA were negatively correlated with IOP values. It is conceivable that osmotic pressure differences caused by hemodialysis may increase lens thickness. The lens thickening leads to a smaller angle opening distance, a narrower trabecular iris angle, and thus increased resistance to aqueous humor drainage. These anatomical modifications are more pronounced in patients with narrow angles.

In recent years, some researchers have also investigated the effects of hemodialysis on anterior chamber parameters. Chong et al. [25] observed changes in anterior chamber parameters before and 2 hours after the end of peritoneal dialysis, including anterior chamber depth, anterior chamber width, anterior chamber area, anterior chamber volume, lens vault, angle opening distance, trabecular iris space area, and angle recess area. Importantly, they reported that there were no statistically significant changes in any of the parameters after dialysis. Caglayan et al. [26] investigated anterior chamber parameters including anterior chamber depth (ACD), aqueous depth (AQD), anterior chamber volume (ACV), and anterior chamber angle (ACA) in 50 eyes before and $30 \mathrm{~min}$ after hemodialysis and reported that there were no significant changes in any of these parameters. This is consistent with the changes in anterior chamber parameters before and after hemodialysis in the present study. In another study, changes in anterior chamber depth were investigated between baseline (the beginning of hemodialysis) and 2 and 4 hours after the start of hemodialysis. In that study, anterior chamber depth was only significantly decreased 2 hours after the start of dialysis [27].

It is known that chronic renal failure patients have insufficient local blood supply to the eyes, and fluctuations in IOP caused by hemodialysis may aggravate this condition or even cause severe irreversible ischemic and hypoxic damage to the optic nerve and retina [28]. Due to the limited anterior chamber angle function of the relevant patients, although the increases in IOP in the extremely narrow-angle group were not sufficient to cause acute high IOP, fluctuation in IOP may affect blood supply to the eye in this particular group of patients with chronic renal failure. In addition, among patients on hemodialysis, there are some with even narrower angles than were observed in the present study and some with complete angle closure. Therefore, it is necessary to improve the screening of high-risk groups before hemodialysis. For example, in patients with cataracts in the initial expansion stage, the lens absorbs water and expands during hemodialysis due to the effects of the osmotic gradient, causing the lens and iris to move forward [20,21], which may lead to relative pupillary block. Aqueous humor drainage is blocked, and IOP increases. In addition, patients with closed-angle glaucoma anatomy (such as small eyeball, 
shallow anterior chamber, and narrow anterior chamber) have drainage problems that lead to increased IOP. In such patients, effective preventive measures such as early cataract surgery or preventive laser peripheral iridotomy should be considered before hemodialysis. High levels of dehydration should also be prevented during hemodialysis. This not only ensures sufficient hemodialysis but also maintains a relatively stable internal environment after each treatment, thereby reducing the occurrence of ocular complications.

In this study, we observed changes in IOP before, during, and after hemodialysis in each group of patients. With respect to anterior chamber parameters, we did not conduct dynamic observation during hemodialysis and thus did not collect anterior chamber-related data when IOP reached its peak, due to procedural limitations, the relative cleanliness of the environment of the dialysis room, the degree of patient cooperation, and the configuration of the equipment, among other reasons. These parameters may recover very rapidly after hemodialysis; so, although they tend to decrease or increase at the end of hemodialysis, the differences are not statistically significant. This does not mean that these relevant parameters did not change during dialysis. The change in IOP after the end of hemodialysis indicates that the increased IOP during hemodialysis in the extremely narrow-angle group had returned to prehemodialysis levels at the end of hemodialysis. Possibly because of the small sample size, there were no cases of complete angle closure in the present study. In future studies, the sample size will be increased, and dynamic changes in anterior parameters during the dialysis process will be investigated to the greatest extent possible.

\section{Abbreviations}

IOP: Intraocular pressure

HD: Hemodialysis

UBM: Ultrasound biomicroscopy

ACD: Central anterior chamber depth

AOD: Angle opening distance at $500 \mu \mathrm{m}$ from scleral spur

TIA: Trabecular iris angle

LT: Lens thickness

IT: Iris thickness

CBT: Ciliary body thickness.

\section{Data Availability}

The datasets used and/or analyzed during the current study are available from the corresponding author on reasonable request.

\section{Ethical Approval}

The study protocol was approved by the Medical Ethics Committee of The Affiliated Hospital of Qingdao University, and the research followed the tenets of the Declaration of Helsinki. Patients who participated in this research signed the informed consent and had complete clinical data.

\section{Consent}

Written consent was obtained from all patients.

\section{Conflicts of Interest}

The authors declare that they have no competing interests.

\section{Authors' Contributions}

WL and WFL participated in the study design. Conduct of the study was contributed by WL, WFL, YZY, CN, and WDB. WFL and CN performed data analysis. WL, WFL, YZY, and CN wrote or contributed to the writing of the manuscript. All authors have read and approved the final manuscript.

\section{Acknowledgments}

We thank colleagues at hemodialysis center in our hospital for providing patients with hemodialysis.

\section{References}

[1] L. C. Lemaitre, B. Bodaghi, N. Cassoux, and P. LeHoang, "Acute choroidal closure caused by hemodialysis accident in an amyloidosic patient," Graefe's Archive for Clinical and Experimental Ophthalmology, vol. 244, no. 6, pp. 758-760, 2006.

[2] W. K. Song, S. J. Ha, H. Y. Yeom, G. J. Seoung, and Y. J. Hong, "Recurrent intraocular pressure elevation during hemodialysis in a patient with neovascular glaucoma," Korean Journal of Ophthalmology, vol. 20, no. 2, pp. 109-112, 2006.

[3] R. A. Burn, "Intraocular pressure during haemodialysis," The British Journal of Ophthalmology, vol. 57, no. 7, pp. 511-513, 1973.

[4] E. Cecchin, M. S. De, and F. Tesio, "Intraocular pressure and hemodialysis," Nephron, vol. 43, no. 1, pp. 73-74, 2004.

[5] C. P. Barbosa, F. R. Stefanini, F. Penha et al., "Intraocular pressure and ocular perfusion during hemodialysis," Arquivos Brasileiros de Oftalmologia, vol. 74, no. 2, pp. 106-109, 2011.

[6] A. Pelit, A. Zümrütdal, and Y. Akova, "The effect of hemodialysis on visual field test in patients with chronic renal failure," Current Eye Research, vol. 26, no. 5, pp. 303-306, 2003.

[7] A. Samsudin, Z. Mimiwati, T. Soong, M. S. Fauzi, and K. Zabri, "Effect of haemodialysis on intraocular pressure," Eye, vol. 24, no. 1, pp. 70-73, 2010.

[8] A. E. Kilavuzoglu, G. Yurteri, N. Guven, S. Marsap, A. R. Celebi, and C. B. Cosar, "The effect of hemodialysis on intraocular pressure," Advances in Clinical and Experimental Medicine, vol. 27, no. 1, pp. 105-110, 2018.

[9] R. N. Shaffer, Stereoscopic manual of gonioscopy, Mosby, St Louis, MO, 1962.

[10] V. Sitprija, J. H. Holmes, and P. P. Ellis, "Changes in intraocular pressure during hemodialysis," Investigative Ophthalmology, vol. 27, pp. 273-284, 1964.

[11] V. Sitprija, J. H. Holmes, and P. P. Ellis, "Intraocular pressure changes during artificial kidney therapy," Archives of Ophthalmology, vol. 72, no. 5, pp. 626-631, 1964. 
[12] M. S. De, E. Cecchin, and F. Tesio, "Intraocular pressure changes during hemodialysis: prevention of excessive dialytic rise and development of severe metabolic acidosis following acetazolamide therapy," Renal Failure, vol. 11, no. 2-3, pp. 117-124, 1989.

[13] R. Hojs and D. Pahor, "Intraocular pressure in chronic renal failure patients treated with maintenance hemodialysis," Ophthalmologica, vol. 211, no. 6, pp. 325-326, 1997.

[14] S. M. Gutmann and N. D. Vaziri, "Effect of hemodialysis on intraocular pressure," Artificial Organs, vol. 8, no. 1, pp. 6265, 1984

[15] U. A. Dinc, S. Ozdek, Z. Aktas, G. Guz, and M. Onol, "Changes in intraocular pressure, and corneal and retinal nerve fiber layer thickness during hemodialysis," International Ophthalmology, vol. 30, no. 4, pp. 337-340, 2010.

[16] S. J. Yang, Y. H. Han, G. I. Song, C. H. Lee, and S. W. Sohn, "Changes of choroidal thickness, intraocular pressure and other optical coherence tomographic parameters after haemodialysis," Clinical \& Experimental Optometry, vol. 96, no. 5, pp. 494-499, 2013.

[17] L. Wang, G. Yin, Z. Y. Yu, N. Chen, and D. Wang, "Effect of hemodialysis on eye coats, axial length, and ocular perfusion pressure in patients with chronic renal failure," Journal of Ophthalmology, vol. 2018, Article ID 3105138, 6 pages, 2018.

[18] A. Albertazzi, B. Di Paolo, C. Spisni, L. Mastropasqua, and P. E. Gallenga, "Intraocular pressure (IOP) changes induced by regular dialysis treatment (RDT)," Life Support Systems, vol. 3, pp. 91-95, 1985

[19] B. Canaud, "Physical principles of renal replacement therapy applied to end stage renal disease patients," Néphrologie \& Thérapeutique, vol. 5, no. 3, pp. 218-238, 2009.

[20] Y. L. Wang, F. Qi, J. L. Xie et al., "Analysis of the relationship between postoperative ophthalmic complications and dialysis time of pre-kidney transplantation," International Journal of Ophthalmology, vol. 5, no. 3, pp. 370-373, 2012.

[21] S. Yin, J. Zhang, X. Hua et al., "Analysis of factors associated with vision after cataract surgery in chronic renal failure patients on dialysis," BMC Ophthalmology, vol. 20, no. 1, pp. 211-221, 2020.

[22] M. Onofriescu, N. G. Mardare, L. Segall et al., "Randomized trial of bioelectrical impedance analysis versus clinical criteria for guiding ultrafiltration in hemodialysis patients: effects on blood pressure, hydration status, and arterial stiffness," International Urology and Nephrology, vol. 44, no. 2, pp. 583-591, 2012.

[23] B. E. Prum Jr., L. W. Herndon Jr., S. E. Moroi et al., "Primary angle closure preferred practice pattern " guidelines," Ophthalmology, vol. 123, no. 1, pp. P1-40, 2016.

[24] J. T. Le, B. Rouse, and G. Gazzard, "Iridotomy to slow progression of visual field loss in angleclosure glaucoma," Cochrane Database of Systematic Reviews, vol. 6, pp. 1-30, 2018.

[25] K. L. Chong, A. Samsudin, T. C. Keng, T. A. Kamalden, and N. Ramli, "Effect of nocturnal intermittent peritoneal dialysis on intraocular pressure and anterior segment optical coherence tomography parameters," Journal of Glaucoma, vol. 26, no. 2, pp. e37-e40, 2017

[26] M. Caglayan, P. Kosekahya, T. Takmaz et al., "Effects of hemodialysis on corneal and anterior chamber morphometry and intraocular pressure in patients with end-stage renal disease," Arquivos Brasileiros de Oftalmologia, vol. 80, no. 3, pp. 176180, 2017
[27] C. P. Gracitelli, F. R. Stefanini, F. Penha et al., "Anterior chamber depth during hemodialysis," Clinical Ophthalmology, vol. 7, pp. 1635-1639, 2013.

[28] W. J. Lee, R. Hong, M. H. Kang et al., "Effect of hemodialysis on peripapillary choroidal thickness measured by sweptsource optical coherence tomography," Journal of Glaucoma, vol. 30, no. 6, pp. 459-464, 2021. 